\title{
Performance of language tasks in patients with ruptured aneurysm of the left hemisphere worses in the post-surgical evaluation
}

\author{
Avaliação da linguagem em pacientes com hemorragia subaracnóidea aneurismática no
} hemisfério esquerdo piora após a cirurgia

Ana Cláudia C. Vieira ${ }^{1}$, Gustavo Andrade ${ }^{2}$, Moysés Ponte de Souza, ${ }^{2}$ Maria de Fátima Leal Griz ${ }^{2}$ Isabel

Eugênia Costa e Silva², Saul M. C. Quinino², Divaldo Câmara Jr², Hildo R.C. Azevedo-Filho²

\begin{abstract}
Sub-arachnoid hemorrhage (SAH) promotes impairment of upper cortical functions. However, few information is available emphasizing changes in language after aneurismal SAH and aneurysm location influence. Objective: To assess the language and verbal fluency performance in aneurismal SAH pre- and post-surgery in patients caused by an aneurysm of the anterior communicating artery (AcomA), left middle cerebral artery (L-MCA) and left posterior comunicating artery (L-PcomA). Methods: Assessment in 79 patients with SAH, on two occasions: pre- and post surgical treatment. They were divided into three groups by the aneurysms' location. Results: Deterioration is detected in the performance of all patients during the post-surgical period; L-MCA aneurysm patients displayed a reduction in verbal naming and fluency; L-PcomA patients deteriorated in the written language and fluency tasks. Conclusion: After the surgical procedure the patients decreased in various language tasks and these differences in performance being directly related to the location of the aneurysm.
\end{abstract}

Keywords: subarachnoid hemorrhage; intracranial aneurysm; language.

\section{RESUMO}

Hemorragia subaracnóidea (HSA) proporciona alterações nas altas funções cerebrais. Contudo, há poucos trabalhos enfatizando as alterações da linguagem na HSA aneurismática e a influência da localização do aneurisma. Objetivos: Analisar e comparar os desempenhos da linguagem e fluência verbal na HSA nos períodos pré e pós-cirúrgicos nos pacientes com aneurismas da comunicante anterior (AcomA), cerebral média (ACME) e comunicante posterior esquerdas (AcomPE). Métodos: A avaliação da linguagem de 79 pacientes com HSA aneurismática foi realizada em duas ocasiões: pré e pós cirurgia. Eles foram divididos em três grupos de acordo com a localização do aneurisma. Resultados: Os grupos apresentaram deterioração na avaliação pós-operatória com diferentes características. 0 grupo da ACME demonstraram deterioração na fluência e nomeação, enquanto o da AcomPE pioraram nos testes de linguagem escrita e fluência. Conclusões: Após o tratamento cirúrgico, os pacientes deterioraram em vários itens de linguagem com diferenças relacionadas à localização do aneurisma.

Palavras-chave: hemorragia subaracnóidea; aneurisma intracraniano; linguagem.

The existence of blood in the subarachnoid space (SAH) is known to trigger disorders of the upper cortical functions ${ }^{1}$. For this reason, researchers have tried to compare the changes detected in aneurismal SAH patients and other SAH patients with no etiology. The results, however, were not conclusive.

Among the SAH cognitive disturbances, memory impairments are the most frequent. Changes in this function cause modifications in the quality of life and the functional activities of persons who, in the majority of cases, are in full productive age $^{2}$. As changes in verbal language, memory, and fluency are more associated with lesions in the left hemisphere, this paper's proposal was to confirm whether these functions already had a different performance in the pre-surgical stage, in the three groups under assessment. In the same manner as there is little literature on aneurysms in the left middle cerebral artery (L-MCA) and left posterior communicating artery (L-PcomA) territory.

The majority of results on cognitive performance analysis involving aneurysms in the AcomA territory display memory

${ }^{1}$ Universidade Federal de Pernambuco, Departamento de Fonoaudiologia, Recife PE, Brasil;

${ }^{2}$ Hospital da Restauração, Departamento de Neurocirurgia, Recife PE, Brasil.

Correspondence: Ana Cláudia de Carvalho Vieira; Rua Dr.João Santos Filho, 250/1001; 52060-615 RecifePE, Brasil; E-mail: ana.vieira.fono@gmail.com

Conflict of interest: There is no conflict of interest to declare.

Received 22 June 2015; Received in final form 05 January 2016; Accepted 02 June 2016. 
and personality changes ${ }^{3,4,5}$. Some phonological and verbal fluency changes have been demonstrated ${ }^{6,7.8}$. Mavaddat and col. showed that longer or shorter time elapsed between a $\mathrm{SAH}$ and the surgical procedure did not influence the results of the neuropsychological tests ${ }^{8}$.

Studies on neuropsychological changes in patients with a middle cerebral artery (MCA) aneurysm are much less described than those found among AcomA aneurysm patients 9 . In 1989, Barbarotto and col. compared the cognitive performance of MCA aneurysm patients and those with an aneurysm of the PcomA. The results demonstrated changes in naming, verbal fluency, and short-term verbal memory in patients with aneurysms on both left-side arteries, and changes in spatial memory on the right side. They concluded that the two groups had similar cognitive profiles ${ }^{10}$.

Haug and col. published two recent papers on cognitive performance of MCA aneurysm patients. In the first paper, they compared cognitive changes in 22 patients with ruptured MCA aneurysms and 15 patients with unruptured aneurysms in the same artery, three months and one year after the treatment. Among these results, the SAH group had greater difficulty in returning to professional activities, and had a reduced verbal memory. However, both groups experienced a reduction in quality of life and in performing cognitive functions ${ }^{11}$. In the second paper, the researchers compared the cognitive profiles of SAH patients from AcomA and MCA aneurysm. These assessments were also performed one year after the SAH. The results showed that there was no significant difference between the groups in language performance, in visual-spatial functions, speed of movements, or attention. However, both groups had a reduced score in these activities when compared to the normal population'.

The difficulty in detecting whether SAH cognitive changes result from losses inherent to the disease or from the procedure selected to occlude the aneurysm are still present. In most neurosurgical centers, treatment is anticipated in order to avoid further bleeding ${ }^{12}$. Nonetheless, the reality of developing countries' health systems does not permit further anticipation in surgery for aneurismal SAH patients. Factors such as difficulties in diagnosing and a precise referral from units located in rural areas, overcrowding in emergency facilities, difficulties on performing tomographic and angiographic exams cause great delays in treatment. For this reason, unfortunately, aneurismal SAH patients at Hospital da Restauração were only submitted to treatment of the ruptured intracranial aneurysm 15 to 20 days following hospitalization.

In the light of our reality and the difficulty in anticipating treatment of ruptured intracranial aneurysms, it was possible to analyze whether the nature of cognitive lesions is a result of hemorrhage itself or a consequence of the process for treating the aneurysms. In our first study 193 patients were assessed before the procedure of, and cognitive impairment was already detected in preoperation period ${ }^{13}$. Hence, this study sought to distinguish the performance of language and verbal fluency in the pre- and post-surgical periods in patients with AcomA and left hemisphere aneurismal SAH.

\section{METHODS}

This prospective study was carried out between May 2007 and November 2009. Excluding posterior circulation aneurysm, patients over 75 years old, Hunt \& Hess $>3$ and associated intraparenchimatous hematoma, we collected 193 patients with anterior circulation aneurysm treated at our institution. Among them, 122 patients were submitted to surgical clipping and were able to complete the cognitive evaluation pre- and post-surgical clipping. From those, only 79 had aneurysm at AcomA (45), L-MCA (19) and L-PComA(15). All of them had diagnostic confirmation by computerized tomography or cerebrospinal fluid examination and digital angiography.

The average age was $47.96 \pm 9.60$ years for women and $45.10 \pm 11.78$ years for men. There were 45 AcomA aneurysms patients, 19 L-MCA, and 15 L-PcomA.

The first assessment of the cognitive functions was performed between 7 and 10 days after de SAH onset, by one of the authors (ACCV) in the Neurosurgery Interdisciplinary Care Ward. After, at least, eight days following the surgical procedure, the patients were re-assessed with the same sequence of cognitive tests.

To begin with, an interview was held with the patient and companion to obtain general data on the SAH background. During this conversation the patient replied questions as occupation, education, the time when the first symptoms appeared, his way to our service, and current status. This interview was intended to confirm the patient's consistency in speech, besides gathering the required data. Soon afterwards, the neuropsychological assessment took place.

The protocols employed were the Montreal-Toulouse Protocol: Aphasia Examination, and initial Standard Module alpha version ${ }^{14}$. This test was employed in Brazil in a multi-center study developed by Lecours and $\mathrm{col}^{14}$. Hence, the protocol shows features that are adequate to our patients reality, such as regional particularities and level of education. The verbal fluency tests embedded in the CERAD ${ }^{15}$ and the Edinburgh inventory for manual dominance ${ }^{16}$ were also applied. All those tests are already described and validated in English literature ${ }^{14,15,16}$.

Following the assessments held in the pre- and post-operative periods, the patients were divided into three groups regarding to the location of the aneurysms, as follows: AcomA, L-MCA and L-PcomA.

The performance in pre and post-surgical language and verbal fluency assessments was compared, and comparison of these results with a control group of individuals who had similar characteristics regarding age and educational skills was also conducted. This group of volunteers was formed by individuals from the actual environment of the patients, 
particularly relatives that presented a similar degree of education and socio-cultural coexistence.

The study was approved by the research ethics committee of Hospital da Restauração, with the final decision documented in protocol CAAE no. 0005.0.102.172-07. To participate in the study, all patients signed the Informed Consent Form.

The data analysis took place with the software program SPSS 13.0 for Windows and Excel 2003. A Kolmogorov-Smirnov normality test was run for quantitative variables. In the independent comparison among the three groups were employed the ANOVA (normal distribution), with multiple comparison of Turkey and Krusskall Wallis (non-normal) and Dunnet's test. The Student Test was employed when distribution was normal and Wilcoxon for non-normal distribution when the groups were analyzed on two occasions (pre- and post-operative) (Confidence Level $=95 \%$, Margin of Error $=5 \%$ ).

\section{RESULTS}

Table 1 reflects the results of language assessment, of verbal fluency and memory in AcomA aneurysm patients during the period before surgery, as compared to the control group. These results showed statistically significant differences in written language tasks, in verbal fluency and memory, with a clear reduction of performance in AcomA aneurysm patients. This difference confirms the presence of serious cognitive changes in these patients, even before being submitted to surgical treatment.

Performance by patients in each task assessed during the pre-surgical period were compared among the groups in order to confirm whether there was a difference in language and verbal fluency profiles determined by aneurysm location (Table 2).

The results of oral language in L-MCA aneurysm patients displayed a larger deficit than those detected in patients with lesions in other sites, with statistically significant differences in repetition and oral naming tasks, confirmed Dunnet's test for multiple comparisons. However, performance in written language activities in AcomA and L-PcomA aneurysm patients was also poor, yet with no differences when compared to L-MCA aneurysms. This also occurred with phonological fluency, which requires knowledge of reading to be evoked.

Following this analysis, performance of the two assessments made in the pre- and post-operative periods were

Table 1. Performance in language tests according to the Montreal-Toulouse Protocol, the CERAD fluency and verbal memory in the pre-operative period of patients with ruptured aneurysms of the ACOA territory, and compared to the control group.

\begin{tabular}{|c|c|c|c|}
\hline \multirow{2}{*}{ Variable } & \multicolumn{2}{|c|}{ Pre-surgery (Mean \pm SD) } & \multirow{2}{*}{$\mathrm{p}$-value } \\
\hline & $\operatorname{AcomA}(n=45)$ & Control $(n=50)$ & \\
\hline Comprehension (maximum score $=11$ ) & $9.49 \pm 1.714$ & $9.66 \pm 1.255$ & $0.9880^{1}$ \\
\hline Repetition (maximum score $=11$ ) & $10.38 \pm 0.684$ & $10.10 \pm 0.814$ & $0.1229^{1}$ \\
\hline Naming (maximum score = 12) & $11.40 \pm 1.514$ & $11.46 \pm 0.613$ & $0.1656^{1}$ \\
\hline Written comprehension (maximum score $=11$ ) & $8.74 \pm 2.748$ & $10.53 \pm 0.772$ & $0.0010 * 1$ \\
\hline Oral reading (maximum score $=11$ ) & $9.09 \pm 2.904$ & $10.89 \pm 0.459$ & $0,0014^{* 1}$ \\
\hline Semantic fluency & $11.51 \pm 4.165$ & $17.00 \pm 4.972$ & $0.0003 * 2$ \\
\hline Phonological fluency & $8.53 \pm 4.494$ & $14.57 \pm 4.669$ & $0.0010 * 2$ \\
\hline Verbal memory & $13.45 \pm 3.785$ & $19.19 \pm 3.720$ & $<0.0001 * 2$ \\
\hline
\end{tabular}

SD: standard deviation; ${ }^{1}$ Mann Whitney test; ${ }^{2}$ T comparative test; ${ }^{\star} p$ value satistically significant differences.

Table 2. Performance in language tests according to the Montreal-Toulouse Protocol and the CERAD Neuropsychological Battery verbal fluency in the pre-operative period of patients with ruptured aneurysms of the AComA, MCA- $L$, and PCOA- $L$ territories.

\begin{tabular}{|c|c|c|c|c|}
\hline \multirow{2}{*}{ Variable } & \multicolumn{3}{|c|}{ Pre-surgery (Mean \pm SD) } & \multirow{2}{*}{$p$-value } \\
\hline & AcomA $(n=45)$ & MCA-L $(n=19)$ & PCOA-L $(n=15)$ & \\
\hline Comprehension (maximum score $=11$ ) & $9.49 \pm 1.714$ & $7.74 \pm 3.297$ & $9.00 \pm 1.773$ & $0.077^{1}$ \\
\hline Repetition (maximum score $=11$ ) & $10.38 \pm 0.684$ & $8.79 \pm 2.760$ & $10.20 \pm 1.821$ & $0.021 * 1$ \\
\hline Naming (maximum score = 12) & $11.40 \pm 1.514$ & $9.26 \pm 4.067$ & $10.47 \pm 2.748$ & $0.007 * 1$ \\
\hline Written comprehension $($ maximum score $=11$ ) & $8.74 \pm 2.748$ & $8.58 \pm 3.343$ & $8.67 \pm 2.060$ & $0.609^{1}$ \\
\hline Oral reading (maximum score $=11$ ) & $9.09 \pm 2.904$ & $9.50 \pm 2.576$ & $9.92 \pm 3.147$ & $0.156^{1}$ \\
\hline Semantic fluency & $11.51 \pm 4.165$ & $9.84 \pm 5.315$ & $10.40 \pm 4.306$ & $0.372^{2}$ \\
\hline Phonological fluency & $8.53 \pm 4.494$ & $8.91 \pm 4.437$ & $7.17 \pm 5.184$ & $0.593^{2}$ \\
\hline
\end{tabular}

SD: standard deviation; ${ }^{1}$ Kruskall Wallis test; ${ }^{2}$ Anova test; * $p$ value satistically significant differences. 
compared, with the purpose of confirming whether the surgical procedure produced cognitive losses for each aneurysm's location. The results are shown in the Table 3.

In Table 3, deterioration is detected in the performance of AcomA aneurysm patients during the post-surgical period, encompassing all language and in the verbal fluency tasks. Yet, statistically significant differences took place in the repetition and semantic fluency tasks only, during the two assessments.

As shown in Table 4, L-MCA post-surgical aneurysm patients displayed a reduction in verbal naming and fluency (semantic and phonological), reflecting in statistically significant differences in the performance of these tasks as compared to the assessments prior to the surgical procedure. It was not possible to measure verbal memory performance in this group due to the small number of patients, and to the fact that evaluation was not performed on all patients. The same occurred with L-PcomA aneurismal SAH patients.

In Table 5, with regard to L-PcomA patients, results also deteriorated following surgery, in all the verbal language and fluency tasks. However, the only task with statistically

Table 3. Performance in language tests according to the Montreal-Toulouse Protocol and the CERAD Neuropsychological Battery verbal fluency in the pre- and post-operative periods of patients $(n=45)$ with ruptured AComA aneurysms.

\begin{tabular}{|c|c|c|c|}
\hline Variables & Pre-surgery (Mean $\pm \mathrm{SD}$ ) & Post-surgery (Mean \pm SD) & $\mathrm{p}$-value \\
\hline Comprehension (maximum score $=11$ ) & $9.49 \pm 1.71$ & $8.93 \pm 2.86$ & $0.069^{1}$ \\
\hline Repetition (maximum score $=11$ ) & $10.38 \pm 0.68$ & $9.71 \pm 2.32$ & $0.027 * 1$ \\
\hline Naming (maximum score $=12$ ) & $11.40 \pm 1.51$ & $10.76 \pm 3.00$ & $0.155^{1}$ \\
\hline Written comprehension $($ maximum score $=11)$ & $8.74 \pm 2.74$ & $8.34 \pm 3.19$ & $0.529^{1}$ \\
\hline Oral reading (maximum score $=11)$ & $9.09 \pm 2.90$ & $8.77 \pm 3.70$ & $0.867^{1}$ \\
\hline Semantic fluency & $11.51 \pm 4.16$ & $9.24 \pm 5.04$ & $0.003 * 2$ \\
\hline Phonological fluency & $8.53 \pm 4.49$ & $7.42 \pm 4.93$ & $0.079^{2}$ \\
\hline
\end{tabular}

SD: standard deviation; ${ }^{1}$ Wilcoxon; ${ }^{2}$ T comparative test; ${ }^{\star} p$ value satistically significant differences.

Table 4. Performance in language tests according to the Montreal-Toulouse Protocol and the CERAD Neuropsychological Battery verbal fluency in the pre- and post-operative periods of patients $(n=19)$ with ruptured MCA- $L$ aneurysms.

\begin{tabular}{lccc}
\hline \multirow{2}{*}{ Variables } & \multicolumn{2}{c}{ Time (Mean \pm SD) } & \multirow{2}{*}{ p-value* } \\
\cline { 2 - 3 } Comprehension (maximum score =11) & Pre-surgery & Post-surgery & $0.750^{1}$ \\
Repetition (maximum score = 11) & $7.74 \pm 3.30$ & $7.79 \pm 2.74$ & $0.842^{1}$ \\
Naming (maximum score = 12) & $8.79 \pm 2.76$ & $8.37 \pm 2.95$ & $0.048^{\star 1}$ \\
Written comprehension (maximum score = 11) & $9.26 \pm 4.07$ & $8.21 \pm 4.80$ & $0.144^{1}$ \\
Oral reading (maximum score =11) & $8.58 \pm 3.34$ & $7.83 \pm 3.24$ & $0.236^{1}$ \\
Semantic fluency & $9.50 \pm 2.58$ & $8.33 \pm 4.14$ & $0.014^{\star 2}$ \\
Phonological fluency & $9.84 \pm 5.32$ & $6.84 \pm 5.16$ & $0.012^{\star 2}$ \\
\hline
\end{tabular}

SD: standard deviation; ${ }^{1}$ Wilcoxon; ${ }^{2}$ T comparative test; ${ }^{\star} p$ value satistically significant differences.

Table 5. Performance in language tests according to the Montreal-Toulouse Protocol and the CERAD Neuropsychological Battery verbal fluency in the pre- and post-operative periods of patients $(n=15)$ with ruptured PCOA-L aneurysms.

\begin{tabular}{|c|c|c|c|}
\hline Variables & Pre-surgery (Mean \pm SD) & Post-surgery (Mean \pm SD) & $p$-value* \\
\hline Comprehension (maximum score $=11$ ) & $9.00 \pm 1.77$ & $8.40 \pm 2.35$ & $0.385^{1}$ \\
\hline Repetition (maximum score $=11$ ) & $10.20 \pm 1.82$ & $9.80 \pm 1.47$ & $0.194^{1}$ \\
\hline Naming (maximum score = 12) & $10.47 \pm 2.75$ & $9.73 \pm 3.06$ & $0.263^{1}$ \\
\hline Written comprehension (maximum score $=11$ ) & $8.67 \pm 2.06$ & $6.75 \pm 3.60$ & $0.014^{\star 1}$ \\
\hline Oral reading (maximum score $=11$ ) & $9.92 \pm 3.15$ & $8.17 \pm 3.86$ & $0.056^{1}$ \\
\hline Semantic fluency & $10.40 \pm 4.31$ & $8.27 \pm 6.42$ & $0.058^{2}$ \\
\hline Phonological fluency & $7.17 \pm 5.18$ & $5.58 \pm 5.95$ & $0.106^{2}$ \\
\hline
\end{tabular}

SD: standard deviation; ${ }^{1}$ Wilcoxon; ${ }^{2} T$ comparative test; ${ }^{*} p$ value satistically significant differences. 
significant differences in performance was written comprehension. It is important to note that in the oral reading and semantic fluency tasks, patients also had a much poorer performance than that shown during the first assessment, yet there were no statistically significant differences.

\section{DISCUSSION}

Patients had different cognitive profiles according to the aneurysm's location, in the pre- and post-surgical periods. Nonetheless, several similarities were detected such as a reduced verbal fluency performance. After surgery, the three groups under assessment had low scores in this ability. Reduced written language tasks were also found in all three groups, with no statistically significant differences among them. Tasks related to read and write learning require a greater cognitive performance and are influenced by abilities found in executive functions, as seen previously ${ }^{17}$. Hence, it is likely that an inter-relation may occur between the functions, resulting in greater cognitive damage.

When comparing the results of the three patient groups, pre- and post-surgical, reduced performance was seen in the cognitive tests in all the groups. However, on analyzing each group according to the aneurysm's location, some differences emerged. Previous papers showed that there is still no definition of the cognitive changes arising from surgical procedures, as it is not possible to have an adequate control group ${ }^{18}$.

Following surgery, AcomA aneurysm patients showed a reduced response to repetition tasks, and statistically significant differences were detected in repetition performance between the results of the two assessments in this group. Initially, this specific task had no relation with the AcomA area, yet surgical access may determine an inability to repeat, as it is made through the Sylvian fissure ${ }^{12,19}$, which is closely related to language. In the survey by Mavaddat et al. ${ }^{8}$, patients who were submitted to surgery to block an AcomA aneurysm had cognitive disorders compatible with a temporal lobe dysfunction. However, a more detailed analysis of the second assessment showed the existence of perseverance in the response to previous stimuli, which cancelled the responses to the following stimuli, besides a great difficulty in retaining the last two test sentences, as they were the longest. These difficulties were not detected in the initial assessment. A difficulty in attention, in working memory, and a reduced inhibition of previous stimuli were the key factors that led to reduced performance in the repetition task by these patients. Hence, we cannot assert that a reduction in the repetition task is related to specific faults in the language function.

Only $15 \mathrm{~L}$-PcomA patients were assessed in the two phases of the study. Nonetheless, this reduced number of patients has shown statistically significant differences in the performance of written comprehension tasks when assessed after surgery. As previously reported, there is very little experience with cognitive damages in patients with L-PcomA aneurysms. There is evidence in this survey of the participation of language functions such as naming in the pre-surgical period as compared to the AcomA group, with written comprehension disorders detected in the two assessment instances. Please note that the reading $(p=0.056)$ and verbal fluency $(p=0.058)$ tasks showed almost significant differences after surgical procedures, and the results may be more evident in a broader sample.

In conclusion, AcomA, L-MCA, and L-PcomA aneurysm patients showed differences in only two language tasks in the period prior to surgery, confirming the assumptions that the aneurysm site could be a determinant factor of cognitive impairment. Other functions such as written language tasks had similar behavior. Surgical clipping added cognitive changes to all three groups of aneurysm location.

\section{References}

1. Sonesson B, Säveland H, Ljunggren B, Brandt L. Cognitive functioning after subarachnoid haemorrhage of unknown origin. Acta Neurol Scand. 1989;80(5):400-10. doi:10.1111/j.1600-0404.1989.tb03901.x

2. Clinchot DM, Bogner JA, Kaplan PE. Cerebral aneurysms: analysis of rehabilitation outcomes. Arch Phys Med Rehabil. 1997;78(4):346-9. doi:10.1016/S0003-9993(97)90223-9

3. Jankowicz E, Halicka D, Drozdowski W. [Neuropsychological deficits after surgical repair of anterior communicating artery aneurysm]. Neurol Neurochir Pol. 2002;36(2):315-27. Polish.

4. Säveland H, Sonesson B, Ljunggren B, Brandt L, Uski T, Zygmunt S et al. Outcome evaluation following subarachnoid hemorrhage.J Neurosurg. 1986;64(2):191-6. doi:10.3171/jns.1986.64.2.0191

5. Sonesson B, Ljunggren B, Säveland H, Brandt L. Cognition and adjustment after late and early operation for ruptured aneurysm. Neurosurgery. 1987;21(3):279-87. doi:10.1227/00006123-198709000-00001

6. Fontanella MM, Bergamasco L, Perozzo P, Priano L, Vighetti S, Griva Fet al. Neuropsychological and neurophysiological evaluation after anterior communicating artery (ACOA) aneurysm surgery. J Neurosurg Sci. 2000;44(2):61-6.
7. Fontanella M, Perozzo P, Ursone R, Garbossa D, Bergui M. Neuropsychological assessment after microsurgical clipping or endovascular treatment for anterior communicating artery aneurysm. Acta Neurochir (Wien). 2003;145(10):867-72. doi:10.1007/s00701-003-0111-5

8. Mavaddat N, Sahakian BJ, Hutchinson PJ, Kirkpatrick PJ. Cognition following subarachnoid hemorrhage from anterior communicating artery aneurysm: relation to timing of surgery. J Neurosurg. 1999;91(3):402-7. doi:10.3171/jns.1999.91.3.0402

9. Haug T, Sorteberg A, Sorteberg W, Lindegaard KF, Lundar T, Finset A. Cognitive functioning and health related quality of life after rupture of an aneurysm on the anterior communicating artery versus middle cerebral artery. Br J Neurosurg. 2009;23(5):507-15. 10.1080/02688690902785701

10. Barbarotto R, De Santis A, Laiacona M, Basso A, Spagnoli D, Capitani E. Neuropsychological follow-up of patients operated for aneurysms of the middle cerebral artery and posterior communicating artery. Cortex. 1989;25(2):275-88. doi:10.1016/S0010-9452(89)80043-7 
11. Haug T, Sorteberg A, Sorteberg W, Lindegaard KF, Lundar T, Finset A. Surgical repair of unruptured and ruptured middle cerebral artery aneurysms: impact on cognitive functioning and health-related quality of life. Neurosurgery. 2009;64(3):412-20. doi:10.1227/01.NEU.0000338952.13880.4E

12. Yun JK, Kang SD, Kim JM. Clipping of the anterior communicating artery aneurysm without Sylvian fissure dissection. J Korean Neurosurg Soc. 2007;42(5):388-91. doi:10.3340/jkns.2007.42.5.388

13. Vieira ACC, Azevedo-Filho HC, Quinino S, Souza MLP, Câmara Jr D, Leitão L et al. Language, memory, and verbal fluency changes in patients with aneurysmal subarachnoid hemorrhage: results of a preoperative investigation. World Neurosurg. 2011;75(5-6):653-9. doi:10.1016/j.wneu.2010.10.044

14. Lecours AR, Mehler J, Parente MA, Caldeira A, Cary L, Castro MJ et al. Illiteracy and brain damage. Aphasia testing in culturally contrasted populations (control subjects). Neuropsychologia. 1987;25(1B):231-45. doi:10.1016/0028-3932(87)90134-5
15. Bertolucci PH, Okamoto IH, Brucki SM, Siviero MO, Toniolo Neto $J$, Ramos LR. Applicability of the CERAD neuropsychological battery to Brazilian elderly. Arq Neuropsiquiatr. 2001;59(3A):532-6. doi:10.1590/S0004-282X2001000400009

16. Oldfield RC. The assessment and analysis of handedness: the Edinburgh inventory. Neuropsychologia. 1971;9(1):97-113. doi:10.1016/0028-3932(71)90067-4

17. Sesma HW, Mahone EM, Levine T, Eason SH, Cutting LE. The contribution of executive skills to reading comprehension. Child Neuropsychol. 2009;15(3):232-46. doi:10.1080/09297040802220029

18. Orozco-Giménez C, Katati MJ, Vilar R, Meersmans M, Pérez-García M, Martín JM et al. [Neuropsychological impairment in patients with intracranial aneurysms: surgical versus endovascular treatment]. Neurocirugia (Astur).Spanish. 2006;17(1):34-44.

19. Stenhouse LM, Knight RG, Longmore BE, Bishara SN. Long-term cognitive deficits in patients after surgery on aneurysms of the anterior communicating artery. J Neurol Neurosurg Psychiatry. 1991;54(10):909-14. doi:10.1136/jnnp.54.10.909 